\title{
Safety and efficacy of abemaciclib plus endocrine therapy in older patients with hormone receptor-positive/human epidermal growth factor receptor 2-negative advanced breast cancer: an age-specific subgroup analysis of MONARCH 2 and 3 trials
}

\author{
Matthew P. Goetz ${ }^{1}$ (D) Meena Okera ${ }^{2} \cdot$ Hans Wildiers $^{3} \cdot$ Mario Campone $^{4} \cdot$ Eva-Maria Grischke $^{5} \cdot$ Luis Manso $^{6}$. \\ Valérie A. M. André ${ }^{7}$. Nadia Chouaki ${ }^{7}$. Belén San Antonio ${ }^{8} \cdot$ Masakazu Toi $^{9} \cdot$ George W. Sledge Jr. $^{10}$
}

Received: 14 September 2020 / Accepted: 16 November 2020 / Published online: 3 January 2021

(C) The Author(s) 2021

\begin{abstract}
Purpose Abemaciclib in combination with endocrine therapy (ET) has demonstrated significant efficacy benefits in HR+, HER2- advanced breast cancer patients in the Phase 3 studies MONARCH 2 (fulvestrant as ET) and MONARCH 3 (letrozole or anastrozole as ET). Here, we report age-specific safety and efficacy outcomes.

Methods Exploratory analyses of MONARCH 2 and 3 were performed for 3 age groups $(<65,65-74$, and $\geq 75$ years). For safety, data were pooled from both studies; for efficacy, a subgroup analysis of PFS was performed for each trial independently.

Results Pooled safety data were available for 1152 patients. Clinically relevant diarrhea (Grade 2/3) was higher in older patients receiving abemaciclib +ET $(<65,39.5 \% ; 65-74,45.2 \% ; \geq 75,55.4 \%)$ versus placebo + ET $(<65,6.8 \% ; 65-74$, $4.5 \% ; \geq 75,16.0 \%)$. Nausea, decreased appetite, and venous thromboembolic events were all moderately higher in older patients. Neutropenia (Grade $\geq 3)$ did not differ as a function of age in the abemaciclib +ET arm $(<65,25.8 \% ; 65-74$, $27.4 \% ; \geq 75,18.1 \%)$. Dose adjustments and discontinuation rates were slightly higher in older patients. Abemaciclib + ET improved PFS compared with placebo + ET independent of patient age, with no significant difference in abemaciclib treatment effect between the 3 age groups (MONARCH 2: interaction $p$-value, 0.695; MONARCH 3: interaction $p$-value, 0.634). Estimated hazard ratios ranged from 0.523-0.633 (MONARCH 2) and 0.480-0.635 (MONARCH 3).

Conclusions While higher rates of adverse events were reported in older patients, they were manageable with dose adjustments and concomitant medication. Importantly, a consistent efficacy benefit was observed across all age groups.

Clinical trial registration ClinicalTrials.gov: NCT02107703 (first posted April 8, 2014) and NCT02246621 (first posted September 23, 2014).
\end{abstract}

Keywords Abemaciclib $\cdot$ Age $\cdot$ HR $+\cdot$ HER2 $-\cdot$ Metastatic breast cancer $\cdot$ Endocrine therapy

Matthew P. Goetz

Goetz.Matthew@mayo.edu

1 Department of Oncology, Mayo Clinic, 200 First St. S.W, Rochester, MN 55905, USA

2 Adelaide Cancer Center, Adelaide, Australia

3 Department of General Medical Oncology, University Hospitals Leuven, Leuven, Belgium

4 Institut de Cancerologie de L'Ouest-René Gauducheau, Saint Herblain, France
5 Women's Hospital, University Hospital Tübingen, Tübingen, Germany

612 de Octubre University Hospital, Madrid, Spain

7 Eli Lilly and Company, Paris, France

8 Eli Lilly and Company, Madrid, Spain

9 Breast Cancer Unit, Kyoto University Hospital, Kyoto University, Kyoto, Japan

10 Stanford University School of Medicine, Stanford, CA, USA 


\section{Introduction}

Breast cancer is the leading cancer diagnosis in women, and a significant proportion of these patients are 65 years of age or older $[1,2]$. In the United States from 2013 to 2017 , the $\geq 65$ age group accounted for almost $45 \%$ of all new breast cancer diagnoses (age 65-74, 25.5\% ; 75-84, $13.6 \% ;>84,5.4 \%)$ and two-thirds of breast cancer deaths (age 65-74, 23.4\%; 75-84, 19.7\%; >84, 17.2\%) [3]. Specifically, hormone receptor-positive $(\mathrm{HR}+)$, human epidermal growth factor receptor 2-negative (HER2-) breast cancer subtype has the highest incidence rate per 100,000 across age groups [4], and the majority of HR+, HER2 - subtypes occur in older women [5]. Older patients tend to have a higher incidence of comorbidities, including vascular, gastrointestinal, metabolic, nutritional, and renal disorders, and increased risk for treatment-related toxicities like gastrointestinal and renal toxicities, which has relevance for quality of life $[2,6,7]$. The underrepresentation of older patients in clinical trials may reflect lower enrollment due to perceived risk increase for treatmentrelated adverse events (TEAEs), and/or limitations placed on eligibility due to comorbidities or prior concomitant medications and potential for drug-drug interactions [8]. These points underscore the need for better information on age-specific efficacy, safety, and drug tolerability, particularly in patients older than 75 years.

Current treatment guidelines support the use of endocrine therapy (ET) in combination with a cyclin-dependent kinase (CDK) 4 and 6 inhibitor for treatment of $\mathrm{HR}+$, HER2 - advanced or metastatic breast cancer [9-12]. Three CDK4 and 6 inhibitors are now FDA and EMA approved in combination with ET as initial endocrinebased therapy or after progression on ET for this indication $[13,14]$. Of these, abemaciclib is approved in combination with letrozole or anastrozole [15] as initial treatment, and in combination with fulvestrant after progression on ET [16]. In MONARCH 3, abemaciclib + ET demonstrated statistically significant and clinically meaningful progression-free survival (PFS) improvement compared to ET alone [median 28.18 vs. 14.76 months; hazard ratio (HR), 0.540; $P=0.000002$ ] [17]. For MONARCH 2, both PFS (median 16.4 vs. 9.3 months; HR, $0.553 ; P<0.001$ ) and OS (median 46.7 vs. 37.3 months; HR, $0.757 ; P=0.01$ ) were longer in the abemaciclib arm compared to placebo $[16,18]$. Abemaciclib was also the first CDK4 and 6 inhibitor with approval as monotherapy in patients with refractory HR+, HER2 - metastatic breast cancer (MONARCH 1) [19].

In a recent safety analysis of MONARCH 2 and 3 [20], the most frequent TEAE in patients taking abemaciclib was diarrhea, with clinically significant diarrhea
(Grade $\geq 2$ ) reported in approximately $43 \%$ of patients, and a median onset of 1 week. Grade 3/4 neutropenia occurred in approximately $25 \%$ of patients receiving abemaciclib across studies. However, abemaciclib discontinuation due to these AEs occurred in $<3 \%$ of patients, indicating these AEs could be successfully managed by dose adjustment and/or use of supportive medication. The safety profile together with the established efficacy of abemaciclib supports a favorable clinical benefit/risk ratio overall in patients with HR+, HER2- advanced breast cancer. Despite this, guidance on the use of abemaciclib in older patient populations is lacking. To address this knowledge gap, here, we report an age-specific analysis of the safety and efficacy of abemaciclib in older patient populations from the Phase 3 studies MONARCH 2 and MONARCH 3.

\section{Methods}

\section{Study design}

Data from the Phase 3 trials MONARCH 2 (NCT02107703) and MONARCH 3 (NCT02246621), which studied abemaciclib in combination with ET in patients with $\mathrm{HR}+$, HER2- ABC were included in this analysis. MONARCH 2 was a global, double-blind, Phase 3 study that included women with HR+, HER2- locally advanced or metastatic breast cancer who experienced disease progression while receiving neoadjuvant or adjuvant $\mathrm{ET}, \leq 12$ months after adjuvant ET, or while receiving first-line ET for ABC [16]. MONARCH 3 included post-menopausal women with HR+, HER2- locoregionally recurrent or metastatic breast cancer who had no prior systemic therapy in the advanced setting [15]. Both studies were reviewed and approved by ethical and institutional review boards. All patients provided written informed consent prior to study enrollment. The trials were conducted in accordance with the 1964 Declaration of Helsinki and its later amendments or comparable ethical standards. Full study design details and eligibility criteria have been published $[15,16]$.

\section{Treatments}

Patients in MONARCH 2 received abemaciclib or placebo, orally, twice daily (BID) and fulvestrant $500 \mathrm{mg}$ by intramuscular injection on days 1 and 15 of Cycle 1 and on day 1 of subsequent 28-day cycles. Patients enrolled at study initiation received abemaciclib at $200 \mathrm{mg}$; however, the protocol was amended after review of safety data and dose reduction rates to reduce starting dose to $150 \mathrm{mg}$ for new patients. Patients who were receiving $200 \mathrm{mg}$ underwent a mandatory dose reduction to $150 \mathrm{mg}$ [16]. Patients in MONARCH 3 received abemaciclib $150 \mathrm{mg}$ orally BID (or placebo) plus 
either anastrozole $1 \mathrm{mg}$ or letrozole $2.5 \mathrm{mg}$, both orally once a day. If either abemaciclib or placebo was discontinued, patients were permitted to continue receiving the ET; if the ET required discontinuation, patients were permitted to continue receiving abemaciclib or placebo.

\section{Outcomes and statistical assessments}

Safety and efficacy of abemaciclib were assessed in 3 age subgroups: $<65,65-74$, and $\geq 75$ years. While patients $>65$ are considered older, the $\geq 75$ group is the most representative of a real-world geriatric population with respect to age. Efficacy was evaluated on the intent-to-treat (ITT) population, and safety was assessed in all patients who received $\geq 1$ dose of the study drug. Adverse events (AEs) were graded according to the National Cancer Institute Common Terminology Criteria for Adverse Events (version 4.0). To assess the impact of age on safety and tolerability, data were pooled across studies and summarized using descriptive statistics. Investigator-assessed PFS was analyzed using the Kaplan-Meier method, and the age subgroup analysis of PFS was performed for each trial independently using a Coxproportional hazard model. Interaction test of age groups by treatment arm was performed at the two-sided 0.05 level.

\section{Results}

\section{Patients}

In MONARCH 2 and MONARCH 3, a total of 1162 patients were randomized to receive treatment. For MONARCH 2, patients were allocated to abemaciclib plus fulvestrant $(n=446)$ or placebo plus fulvestrant $(n=223)$
[16]. At study initiation, patients in the abemaciclib arm received a $200 \mathrm{mg}$ BID dose $(n=121,27.4 \%)$ for a median duration of 34 days before a dose reduction to $150 \mathrm{mg}$ or discontinuation. For MONARCH 3, patients were allocated to abemaciclib $150 \mathrm{mg}$ BID plus nonsteroidal AI (either anastrozole or letrozole) $(n=328)$ or placebo plus AI $(n=165)$ (Fig. 1).

Treatment arms for each study were divided into 3 age subgroups: $<65,65-74$, and $\geq 75$ years of age. The ITT population age breakdown of MONARCH 2 was 291 (65.2\%) patients $<65$ years, $114(25.6 \%) 65-74$ years, and $41(9.2 \%) \geq 75$ years of age in the abemaciclib plus fulvestrant arm, and $133(59.6 \%)$ patients $<65$ years, $60(26.9 \%)$ $65-74$ years, and $30(13.5 \%) \geq 75$ years of age in the placebo arm. The ITT population of MONARCH 3 comprised $180(54.9 \%)$ patients $<65$ years, $106(32.3 \%) 65-74$ years, and $42(12.8 \%) \geq 75$ years of age in the abemaciclib plus nonsteroidal AI arm, and 91 (55.2\%) patients $<65$ years, $54(32.7 \%) 65-74$ years, and $20(12.1 \%) \geq 75$ years of age in the placebo arm (Fig. 1).

Distribution of patient baseline disease characteristics was generally well balanced for shared characteristics between the 2 studies, across treatment arms, and between age groups (Table 1, MONARCH 2 and Table 2, MONARCH 3). In both MONARCH 2 and 3, the proportion of patients with an ECOG PS of 1 was generally higher in the 65-74 and $\geq 75$ age groups compared to the $<65$ age group. Older patients had a higher incidence at baseline of comorbidities related to vascular disorders (mainly hypertension), gastrointestinal disorders (constipation, gastroesophageal reflux disease), cardiac disorders, and metabolism and nutrition disorders (including hypercholesterolemia and hyperglycemia).
Fig. 1 Study design. Data cutoff for MONARCH 2 was Feb 14, 2017; MONARCH 3 data cutoff was Nov 3, 2017. BID twice daily, $E T$ endocrine therapy, $H R+$ hormone receptor-positive, HER2- human epidermal growth factor receptor 2 negative, ITT intent to treat, $M 2$ MONARCH 2, M3 MONARCH $3, n$ number of patients, $N$ total population

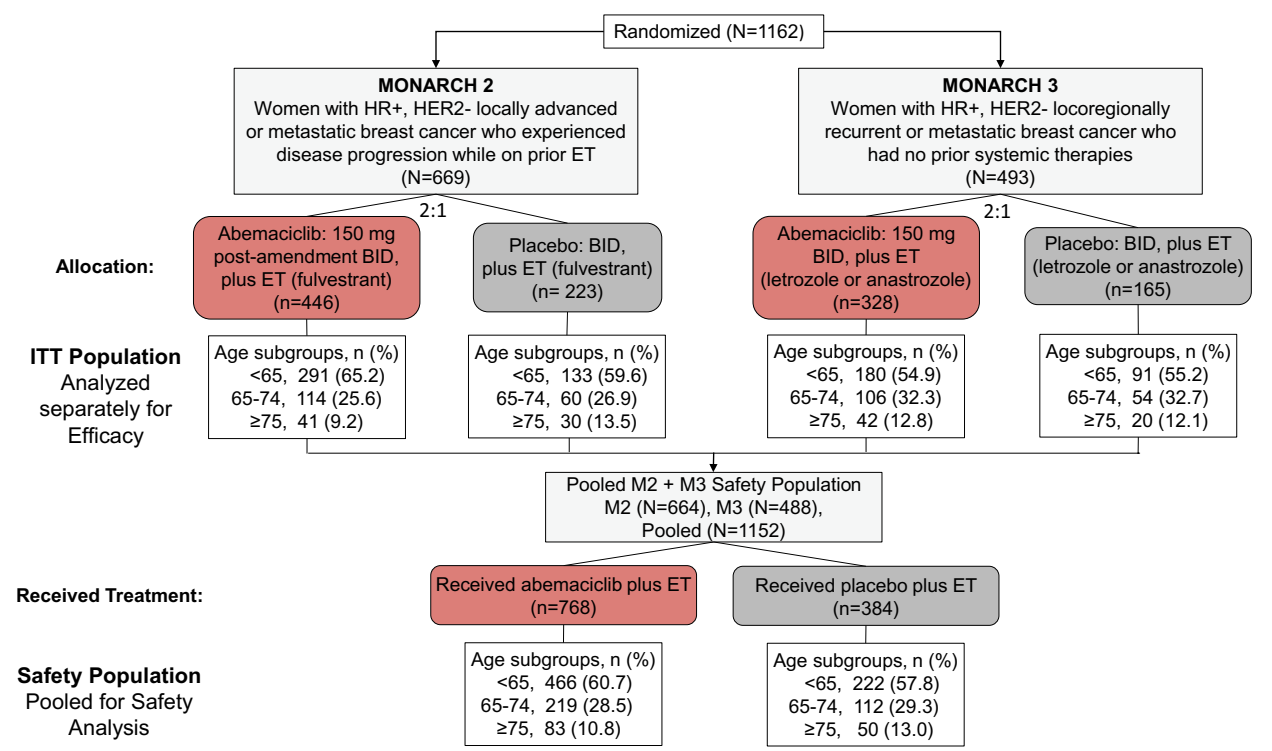


Table 1 Baseline disease characteristics and comorbidities in MONARCH 2 by age subgroup

\begin{tabular}{|c|c|c|c|c|c|c|}
\hline \multirow[t]{3}{*}{ Baseline characteristics, $n(\%)$} & \multicolumn{3}{|c|}{ Abemaciclib $+\mathrm{ET}^{\mathrm{a}}$} & \multicolumn{3}{|c|}{ Placebo $+\mathrm{ET}^{\mathrm{a}}$} \\
\hline & $<65$ & $65-74$ & $\geq 75$ & $<65$ & $65-74$ & $\geq 75$ \\
\hline & $n=291$ & $n=114$ & $n=41$ & $n=133$ & $n=60$ & $n=30$ \\
\hline \multicolumn{7}{|l|}{ ECOG PS $^{b}$} \\
\hline 0 & $182(62.5)$ & $64(56.1)$ & $18(43.9)$ & $88(66.2)$ & $30(50.0)$ & $18(60.0)$ \\
\hline 1 & $104(35.7)$ & $49(43.0)$ & $23(56.1)$ & $45(33.8)$ & $30(50.0)$ & $12(40.0)$ \\
\hline \multicolumn{7}{|l|}{ Nature of the disease } \\
\hline Visceral & $162(55.7)$ & $56(49.1)$ & $27(65.9)$ & $73(54.9)$ & $36(60.0)$ & $19(63.3)$ \\
\hline Bone only & $83(28.5)$ & $32(28.1)$ & $8(19.5)$ & $39(29.3)$ & $13(21.7)$ & $5(16.7)$ \\
\hline Other & $43(14.8)$ & $26(22.8)$ & $6(14.6)$ & $21(15.8)$ & $11(18.3)$ & $6(20.0)$ \\
\hline \multicolumn{7}{|l|}{ PgR status $^{c}$} \\
\hline Positive & $229(78.7)$ & $82(71.9)$ & $28(68.3)$ & $114(85.7)$ & $38(63.3)$ & $19(63.3)$ \\
\hline Negative & 55 (18.9) & $29(25.4)$ & $12(29.3)$ & $18(13.5)$ & $17(28.3)$ & $9(30.0)$ \\
\hline \multicolumn{7}{|l|}{ ET sensitivity } \\
\hline No prior ET & $5(1.7)$ & $1(0.9)$ & $0(0.0)$ & $1(0.8)$ & $1(1.7)$ & $0(0.0)$ \\
\hline Primary resistance & $84(28.9)$ & $20(17.5)$ & $7(17.1)$ & $38(28.6)$ & $13(21.7)$ & $7(23.3)$ \\
\hline Secondary resistance & $199(68.4)$ & $93(81.6)$ & $34(82.9)$ & $94(70.7)$ & $46(76.7)$ & $23(76.7)$ \\
\hline Comorbidities, $n(\%)$ & $n=287$ & $n=113$ & $n=41$ & $n=133$ & $n=60$ & $n=30$ \\
\hline Vascular disorders ${ }^{\mathrm{d}}$ & $105(36.6)$ & $64(56.6)$ & $29(70.7)$ & $51(38.3)$ & $38(63.3)$ & $25(83.3)$ \\
\hline Hypertension & $72(25.1)$ & $60(53.1)$ & $27(65.9)$ & $38(28.6)$ & $35(58.3)$ & $25(83.3)$ \\
\hline Gastrointestinal disorders & $73(25.4)$ & $33(29.2)$ & $17(41.5)$ & $31(23.3)$ & $23(38.3)$ & $12(40.0)$ \\
\hline Constipation & $21(7.3)$ & $10(8.8)$ & $10(24.4)$ & $7(5.3)$ & $4(6.7)$ & $2(6.7)$ \\
\hline Gastroesophageal reflux disease & $13(4.5)$ & $13(11.5)$ & $5(12.2)$ & $7(5.3)$ & $4(6.7)$ & $5(16.7)$ \\
\hline Nausea & $16(5.6)$ & $2(1.8)$ & $2(4.9)$ & $6(4.5)$ & $3(5.0)$ & $1(3.3)$ \\
\hline Abdominal pain & $8(2.8)$ & $5(4.4)$ & $0(0.0)$ & $3(2.3)$ & $1(1.7)$ & $1(3.3)$ \\
\hline Diarrhea & $6(2.1)$ & $8(7.1)$ & $0(0.0)$ & $3(2.3)$ & $4(6.7)$ & $0(0.0)$ \\
\hline Metabolism and nutrition disorders & $57(19.9)$ & $44(38.9)$ & $18(43.9)$ & $29(21.8)$ & $23(38.3)$ & $14(46.7)$ \\
\hline Hypercholesterolemia & $24(8.4)$ & $25(22.1)$ & $13(31.7)$ & $11(8.3)$ & $12(20.0)$ & $6(20.0)$ \\
\hline Hyperglycemia & $14(4.9)$ & $16(14.2)$ & $3(7.3)$ & $10(7.5)$ & $8(13.3)$ & $4(13.3)$ \\
\hline Decreased appetite & $10(3.5)$ & $3(2.7)$ & $0(0.0)$ & $3(2.3)$ & $4(6.7)$ & $1(3.3)$ \\
\hline Cardiac disorders & $26(9.1)$ & $11(9.7)$ & $9(22.0)$ & $14(10.5)$ & $14(23.3)$ & $3(10.0)$ \\
\hline Renal and urinary disorders & $13(4.5)$ & $7(6.2)$ & $3(7.3)$ & $11(8.3)$ & 7 (11.7) & $4(13.3)$ \\
\hline Blood creatinine increase & $3(1.0)$ & $4(3.5)$ & $0(0.0)$ & $0(0.0)$ & $0(0.0)$ & $1(3.3)$ \\
\hline
\end{tabular}

Data given as $n(\%)$ unless otherwise indicated

ECOG PS Eastern Cooperative Oncology Group performance status, ET endocrine therapy, $n$ number of patients, $P g R$ progesterone receptor

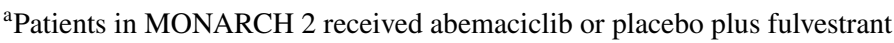

${ }^{\mathrm{b}}$ One patient in the age 65-74 abemaciclib arm had an ECOG PS of 2+

${ }^{c}$ Patients with unknown PgR status not shown

${ }^{\mathrm{d}}$ Embolism reported in $\leq 2.1 \%$ of patients in each age group

\section{Safety}

Pooled safety data were available for 1152 patients treated in the MONARCH 2 and 3 trials (MONARCH 2, N=664; MONARCH 3, $N=488$ ) (Fig. 1). Pooled across treatment arms, this included $688(59.7 \%)<65 ; 331$ (28.7\%) 65-74; and $133(11.5 \%) \geq 75$ years.

The most common TEAEs in the abemaciclib + ET arm (any age group) from the pooled analysis of MONARCH 2 and MONARCH 3 are shown in Table 3. The most frequent any grade TEAE was diarrhea, followed by neutropenia, nausea, and fatigue. The incidence of diarrhea was similar in abemaciclib-treated patients across all age groups $(\sim 85 \%)$. In the abemaciclib arm, other gastrointestinal toxicities such as nausea and decreased appetite were moderately higher (by 10-20\%) in the 2 older subgroups. In contrast, abdominal pain, vomiting, and constipation were not increased in the older subgroups compared to $<65$ group. Fatigue was 
Table 2 Baseline disease characteristics and comorbidities in MONARCH 3 by age subgroup

\begin{tabular}{|c|c|c|c|c|c|c|}
\hline \multirow[t]{3}{*}{ Baseline characteristics, $n(\%)$} & \multicolumn{3}{|c|}{ Abemaciclib $+\mathrm{ET}^{\mathrm{a}}$} & \multicolumn{3}{|c|}{ Placebo $+\mathrm{ET}^{\mathrm{a}}$} \\
\hline & $<65$ & $65-74$ & $\geq 75$ & $<65$ & $65-74$ & $\geq 75$ \\
\hline & $n=180$ & $n=106$ & $n=42$ & $n=91$ & $n=54$ & $n=20$ \\
\hline \multicolumn{7}{|l|}{ ECOG PS } \\
\hline 0 & $115(63.9)$ & $62(58.5)$ & $15(35.7)$ & $60(65.9)$ & $34(63.0)$ & $10(50.0)$ \\
\hline 1 & $65(36.1)$ & $44(41.5)$ & $27(64.3)$ & $31(34.1)$ & $20(37.0)$ & $10(50.0)$ \\
\hline \multicolumn{7}{|l|}{ Disease setting } \\
\hline De novo metastatic & $73(40.6)$ & $42(39.6)$ & $20(47.6)$ & $28(30.8)$ & $25(46.3)$ & $8(40.0)$ \\
\hline Metastatic recurrent & $104(57.8)$ & $58(54.7)$ & $20(47.6)$ & $59(64.8)$ & $29(53.7)$ & $11(55.0)$ \\
\hline Locoregionally recurrent & $3(1.7)$ & $6(5.7)$ & $2(4.8)$ & $4(4.4)$ & $0(0.0)$ & $1(5.0)$ \\
\hline \multicolumn{7}{|l|}{ Nature of the disease } \\
\hline Visceral & $95(52.8)$ & $54(50.9)$ & $23(54.8)$ & 47 (51.6) & $31(57.4)$ & $11(55.0)$ \\
\hline Bone only & $37(20.6)$ & $27(25.5)$ & $6(14.3)$ & $25(27.5)$ & $14(25.9)$ & $0(0.0)$ \\
\hline Other & $48(26.7)$ & $25(23.6)$ & $13(31.0)$ & $19(20.9)$ & $9(16.7)$ & $9(45.0)$ \\
\hline \multicolumn{7}{|l|}{ PgR status ${ }^{b}$} \\
\hline Positive & $139(77.2)$ & $80(75.5)$ & $36(85.7)$ & $71(78.0)$ & $40(74.1)$ & $16(80.0)$ \\
\hline Negative & $41(22.8)$ & $23(21.7)$ & $6(14.3)$ & $20(22.0)$ & $12(22.2)$ & $4(20.0)$ \\
\hline Comorbidities, $n(\%)$ & $n=179$ & $n=106$ & $n=42$ & $n=89$ & $n=52$ & $n=20$ \\
\hline Vascular disorders $^{c}$ & $52(29.1)$ & $61(57.5)$ & $27(64.3)$ & $22(24.7)$ & $27(51.9)$ & $12(60.0)$ \\
\hline Hypertension & $37(20.7)$ & $57(53.8)$ & $25(59.5)$ & $19(21.3)$ & $21(40.4)$ & $12(60.0)$ \\
\hline Gastrointestinal disorders & $33(18.4)$ & $31(29.2)$ & $15(35.7)$ & $20(22.5)$ & $17(32.7)$ & $6(30.0)$ \\
\hline Constipation & $7(3.9)$ & $10(9.4)$ & $3(7.1)$ & $5(5.6)$ & $5(9.6)$ & $1(5.0)$ \\
\hline Gastroesophageal reflux disease & $4(2.2)$ & $7(6.6)$ & $6(14.3)$ & $7(7.9)$ & $4(7.7)$ & $2(10.0)$ \\
\hline Nausea & $7(3.9)$ & $4(3.8)$ & $4(9.5)$ & $3(3.4)$ & $1(1.9)$ & $0(0.0)$ \\
\hline Abdominal pain & $4(2.2)$ & $0(0.0)$ & $0(0.0)$ & $1(1.1)$ & $0(0.0)$ & $0(0.0)$ \\
\hline Diarrhea & $1(0.6)$ & $3(2.8)$ & $0(0.0)$ & $0(0.0)$ & $1(1.9)$ & $0(0.0)$ \\
\hline Metabolism and nutrition disorders & $40(22.3)$ & $55(51.9)$ & $17(40.5)$ & $21(23.6)$ & $21(40.4)$ & $11(55.0)$ \\
\hline Hypercholesterolemia & $16(8.9)$ & $26(24.5)$ & $9(21.4)$ & $5(5.6)$ & $10(19.2)$ & $5(25.0)$ \\
\hline Hyperglycemia & $13(7.3)$ & $21(19.8)$ & $10(23.8)$ & $7(7.9)$ & $7(13.5)$ & $4(20.0)$ \\
\hline Decreased appetite & $3(1.7)$ & $3(2.8)$ & $2(4.8)$ & $3(3.4)$ & $2(3.8)$ & $1(5.0)$ \\
\hline Cardiac disorders & $6(3.4)$ & $11(10.4)$ & $11(26.2)$ & $2(2.2)$ & $8(15.4)$ & $4(20.0)$ \\
\hline Renal and urinary disorders & $7(3.9)$ & $8(7.5)$ & $5(11.9)$ & $5(5.6)$ & $6(11.5)$ & $2(10.0)$ \\
\hline Blood creatinine increase & $0(0.0)$ & $2(1.9)$ & $2(4.8)$ & $0(0.0)$ & $0(0.0)$ & $0(0.0)$ \\
\hline
\end{tabular}

Data given as $n(\%)$ unless otherwise indicated

ECOG PS Eastern Cooperative Oncology Group performance status, ET endocrine therapy, $n$ number of patients, $P g R$ progesterone receptor

${ }^{\text {a } P a t i e n t s ~ i n ~ M O N A R C H ~} 3$ received abemaciclib or placebo plus either letrozole or anastrozole

${ }^{b}$ Patients with unknown PgR status not shown

${ }^{\mathrm{c}}$ Embolism reported in $\leq 3.2 \%$ of patients in each age group

slightly higher in the 2 abemaciclib-treated older age groups $(<65,34.8 \% ; 65-74,48.4 \%, \geq 75,51.8 \%)$. Hematological toxicities including neutropenia, anemia, and leukopenia were higher in the abemaciclib + ET arm compared to the placebo arm, but no differences in incidence were seen across age groups. Increased blood creatinine was higher in the abemaciclib arm and was also higher in both the 65-74 and $\geq 75$ age groups compared to the $<65$ group.

Select AEs of clinical interest are reported in more detail in Table 4. Clinically relevant diarrhea (Grade 2/3) occurred more frequently in the 2 older groups $(<65,39.5 \%$; $65-74$,
$45.2 \% ; \geq 75,55.4 \%)$. Of note, in the placebo arm, Grade $2 / 3$ diarrhea was more common in the $\geq 75$ group compared to the $<65$ group $(<65,6.8 \% ; 65-74,4.5 \% ; \geq 75,16.0 \%)$. Neutropenia was the most common Grade $\geq 3$ AE (Table 4); however, incidence of Grade $\geq 3$ neutropenia did not differ as a function of age in either the abemaciclib arm $(<65,25.8 \%$; $65-74,27.4 \% ; \geq 75,18.1 \%$ ) or placebo arm. Hepatic events (increased ALT, AST, ALP, and bilirubin levels) in the abemaciclib arm were reported with similar, or lower, incidence in the 65-74 and $\geq 75$ subgroups compared to the $<65$ age group. Interstitial lung disease (ILD)/pneumonitis was 
Table 3 Pooled TEAEs from MONARCH 2 and MONARCH

3 occurring in $\geq 20 \%$ patients in any age group in the abemaciclib + ET arm

\begin{tabular}{|c|c|c|c|c|c|c|}
\hline \multirow[t]{2}{*}{ TEAE, any grade, $\mathrm{n}(\%)^{\mathrm{b}}$} & \multicolumn{3}{|c|}{ Abemaciclib $+\mathrm{ET}^{\mathrm{a}}$} & \multicolumn{3}{|c|}{ Placebo $+\mathrm{ET}^{\mathrm{a}}$} \\
\hline & $\begin{array}{l}<65 \\
(n=466)\end{array}$ & $\begin{array}{l}65-74 \\
(n=219)\end{array}$ & $\begin{array}{l}\geq 75 \\
(n=83)\end{array}$ & $\begin{array}{l}<65 \\
(n=222)\end{array}$ & $\begin{array}{l}65-74 \\
(n=112)\end{array}$ & $\begin{array}{l}\geq 75 \\
(n=50)\end{array}$ \\
\hline Diarrhea & $396(85.0)$ & $183(83.6)$ & $71(85.5)$ & $57(25.7)$ & $33(29.5)$ & $17(34.0)$ \\
\hline Neutropenia & $215(46.1)$ & $106(48.4)$ & $25(30.1)$ & $8(3.6)$ & $4(3.6)$ & $0(0.0)$ \\
\hline Nausea & $181(38.8)$ & $114(52.1)$ & $39(47.0)$ & $50(22.5)$ & $24(21.4)$ & $10(20.0)$ \\
\hline Fatigue & $162(34.8)$ & $106(48.4)$ & $43(51.8)$ & $66(29.7)$ & $34(30.4)$ & $14(28.0)$ \\
\hline Abdominal pain & $169(36.3)$ & 67 (30.6) & $22(26.5)$ & $38(17.1)$ & $11(9.8)$ & $7(14.0)$ \\
\hline Anemia & $127(27.3)$ & $79(36.1)$ & $25(30.1)$ & $8(3.6)$ & $9(8.0)$ & $4(8.0)$ \\
\hline Vomiting & $124(26.6)$ & $63(28.8)$ & $26(31.3)$ & $26(11.7)$ & $16(14.3)$ & $2(4.0)$ \\
\hline Decreased appetite & $95(20.4)$ & $75(34.2)$ & $33(39.8)$ & $26(11.7)$ & 13 (11.6) & $5(10.0)$ \\
\hline Leukopenia & $119(25.5)$ & $58(26.5)$ & $20(24.1)$ & $7(3.2)$ & $1(0.9)$ & $0(0.0)$ \\
\hline Alopecia & $102(21.9)$ & $43(19.6)$ & $14(16.9)$ & $14(6.3)$ & $7(6.3)$ & $1(2.0)$ \\
\hline Headache & $109(23.4)$ & $37(16.9)$ & $8(9.6)$ & $50(22.5)$ & $9(8.0)$ & $1(2.0)$ \\
\hline Blood creatinine increase & $42(9.0)$ & $59(26.9)$ & $18(21.7)$ & $2(0.9)$ & $4(3.6)$ & $2(4.0)$ \\
\hline Constipation & $59(12.7)$ & $45(20.5)$ & $13(15.7)$ & $29(13.1)$ & $17(15.2)$ & $7(14.0)$ \\
\hline Cough & $49(10.5)$ & 47 (21.5) & $11(13.3)$ & $28(12.6)$ & $14(12.5)$ & $3(6.0)$ \\
\hline Dyspnea & $36(7.7)$ & $35(16.0)$ & $17(20.5)$ & $20(9.0)$ & $11(9.8)$ & $5(10.0)$ \\
\hline
\end{tabular}

TEAE treatment-emergent adverse event, ET endocrine therapy, $n$ number of patients

${ }^{a}$ Patients in MONARCH 2 received abemaciclib plus fulvestrant; patients in MONARCH 3 received abemaciclib plus either letrozole or anastrozole

${ }^{\mathrm{b}}$ Ordered by decreasing frequency (any grade) of combined age groups (total) in the abemaciclib + ET arm

${ }^{\mathrm{c}}$ There were no Grade 4 diarrhea events infrequent and reported at similar frequency $(\sim 3.5 \%)$ across age groups in the abemaciclib arm, with $<1.5 \%$ of cases Grade $\geq 3$ (Table 4). The incidence of venous thromboembolic events (VTEs), including pulmonary embolism or deep vein thrombosis (DVT), was consistent in the 2 younger age groups $(<65 ; 4.1 \%, 65-74 ; 5.0 \%)$; in contrast, the incidence of VTEs was more common in patients aged $\geq 75(\geq 75 ; 11$ of 83 patients, $13.3 \%$ ).

In the pooled analysis of MONARCH 2 and MONARCH 3, patients treated with abemaciclib in the 65-74 and $\geq 75$ subgroups had more dose adjustments compared to patients $<65$ years of age (Table 5). Abemaciclib dose omissions and reductions were mainly due to AEs. The most frequent AEs leading to dose adjustments were diarrhea and neutropenia. The incidence of dose omissions due to the AE of diarrhea was slightly higher in the 2 older age groups $(<65,12.7 \%$; 65-74, 22.4\%; $\geq 75,30.1 \%)$. However, there was only a modest increase in dose reductions due to the $\mathrm{AE}$ of diarrhea in those groups as compared to the $<65$ patients ( $<65,14.6 \% ; 65-74,18.7 \% ; \geq 75,22.9 \%)$.

Discontinuation of all study treatment due to AEs was higher in the 65-74 and $\geq 75$ subgroups compared to the $<65$ group (Table 5) $(<65,8.8 \%$; 65-74, 14.2\%; $\geq 75,24.1 \%)$. The most common AE leading to study treatment discontinuation in all age groups combined was diarrhea $(n=10$, $1.3 \%$ ), followed by lung infection and ALT increased, which accounted for a small percentage of patients (Table 5). Of these, only diarrhea as the reason for study discontinuation was notably higher in the 2 older subgroups $(<65,0.4 \%$; $65-74,1.8 \% ; \geq 75,4.8 \%$ ) (Table 5). Neutropenia was not a cause for study treatment discontinuation for any patient in either the $65-74$ or $\geq 75$ age group, and only accounted for 3 patients $(0.6 \%)$ discontinuing study treatment in the $<65$ age group.

In MONARCH 2, the age breakdown of patients initially receiving the $200 \mathrm{mg}$ BID dose in the abemaciclib arm was $77(26.5 \%)$ in the $<65$ age group, 30 (26.3\%) 65-74, and $14(34.1 \%) \geq 75$ years. Patients starting with the $200 \mathrm{mg}$ BID dose required more dose adjustments due to AEs in all age groups. The percentage of patients with dose reductions due to AEs starting at $200 \mathrm{mg}$ BID abemaciclib by age group was $<65,51.9 \% ; 65-74,60.0 \%$; $\geq 75,71.4 \%$, compared to patients starting at $150 \mathrm{mg}$ abemaciclib $<65$, $32.4 \%$; 65-74, 50.6\%; $\geq 75,40.7 \%$. Dose reductions due to diarrhea occurred more frequently in MONARCH 2 patients starting on $200 \mathrm{mg}$ BID abemaciclib $(<65,27.3 \%$; $65-74,33.3 \% ; \geq 75,50.0 \%)$ compared to those receiving $150 \mathrm{mg}(<65,11.9 \%$; 65-74, 20.5\%; $\geq 75,11.1 \%)$. In addition, discontinuation of all study treatment due to AEs was also skewed with more cases occurring in the MONARCH 2 patients starting at the $200 \mathrm{mg}$ BID dose $(<65$, $13.0 \% ; 65-74,16.7 \%, \geq 75,21.4 \%$ ) versus in those starting at the $150 \mathrm{mg}$ dose $(<65,4.8 \% ; 65-74,6.0 \% ; \geq 75,18.5 \%)$. Management of AEs was accomplished primarily through 
Table 4 Selected AEs pooled from MONARCH 2 and MONARCH 3

\begin{tabular}{|c|c|c|c|c|c|c|}
\hline \multirow[t]{2}{*}{ AE, $n(\%)$} & \multicolumn{3}{|c|}{ Abemaciclib $+\mathrm{ET}^{\mathrm{a}}$} & \multicolumn{3}{|c|}{ Placebo $+E^{a}$} \\
\hline & $\begin{array}{l}<65 \\
(n=466)\end{array}$ & $\begin{array}{l}65-74 \\
(n=219)\end{array}$ & $\begin{array}{l}\geq 75 \\
(n=83)\end{array}$ & $\begin{array}{l}<65 \\
(n=222)\end{array}$ & $\begin{array}{l}65-74 \\
(n=112)\end{array}$ & $\begin{array}{l}\geq 75 \\
(n=50)\end{array}$ \\
\hline \multicolumn{7}{|l|}{ Diarrhea } \\
\hline Any grade & $396(85.0)$ & $183(83.6)$ & $71(85.5)$ & $57(25.7)$ & $33(29.5)$ & $17(34.0)$ \\
\hline Grade $2 / 3$ & $184(39.5)$ & $99(45.2)$ & $46(55.4)$ & $15(6.8)$ & $5(4.5)$ & $8(16.0)$ \\
\hline Grade $3^{\mathrm{b}}$ & $46(9.9)$ & $28(12.8)$ & $16(19.3)$ & $1(0.5)$ & $0(0.0)$ & $2(4.0)$ \\
\hline \multicolumn{7}{|l|}{ Neutropenia } \\
\hline Any grade & $215(46.1)$ & $106(48.4)$ & $25(30.1)$ & $8(3.6)$ & $4(3.6)$ & $0(0.0)$ \\
\hline Grade $\geq 3$ & $120(25.8)$ & $60(27.4)$ & $15(18.1)$ & $4(1.8)$ & $2(1.8)$ & $0(0.0)$ \\
\hline \multicolumn{7}{|c|}{ ALT increase } \\
\hline Any grade & $76(16.3)$ & $33(15.1)$ & $7(8.4)$ & $15(6.8)$ & $6(5.4)$ & $3(6.0)$ \\
\hline Grade $\geq 3$ & $23(4.9)$ & $12(5.5)$ & $4(4.8)$ & $5(2.3)$ & $1(0.9)$ & $1(2.0)$ \\
\hline \multicolumn{7}{|l|}{ AST increase } \\
\hline Any grade & $68(14.6)$ & $34(15.5)$ & $7(8.4)$ & $17(7.7)$ & $7(6.3)$ & $3(6.0)$ \\
\hline Grade $\geq 3$ & $13(2.8)$ & $7(3.2)$ & $2(2.4)$ & $4(1.8)$ & $3(2.7)$ & $1(2.0)$ \\
\hline \multicolumn{7}{|c|}{ Blood ALP increase } \\
\hline Any grade & $20(4.3)$ & $15(6.8)$ & $4(4.8)$ & $8(3.6)$ & $3(2.7)$ & $2(4.0)$ \\
\hline Grade $\geq 3$ & $30.6)$ & $3(1.4)$ & $1(1.2)$ & $0(0.0)$ & $0(0.0)$ & $1(2.0)$ \\
\hline \multicolumn{7}{|c|}{ Blood bilirubin increase } \\
\hline Any grade & $7(1.5)$ & $5(2.3)$ & $1(1.2)$ & $2(0.9)$ & $1(0.9)$ & $0(0.0)$ \\
\hline Grade $\geq 3$ & $5(1.1)$ & $2(0.9)$ & $0(0.0)$ & $0(0.0)$ & $0(0.0)$ & $0(0.0)$ \\
\hline \multicolumn{7}{|l|}{ VTE events ${ }^{c}$} \\
\hline Any grade & $19(4.1)$ & $11(5.0)$ & $11(13.3)$ & $1(0.5)$ & $1(0.9)$ & $1(2.0)$ \\
\hline Grade $\geq 3$ & $9(1.9)$ & $6(2.7)$ & $4(4.8)$ & $1(0.5)$ & $1(0.9)$ & $0(0.0)$ \\
\hline \multicolumn{7}{|c|}{ ILD/pneumonitis events ${ }^{\mathrm{d}, \mathrm{e}}$} \\
\hline Any grade & $16(3.4)$ & $7(3.2)$ & $3(3.6)$ & $2(0.9)$ & $0(0.0)$ & $0(0.0)$ \\
\hline Grade $\geq 3$ & $4(0.9)$ & $3(1.4)$ & $0(0.0)$ & $0(0.0)$ & $0(0.0)$ & $0(0.0)$ \\
\hline
\end{tabular}

$A E$ adverse event, $A L P$ alkaline phosphatase, $A L T$ alanine aminotransferase, $A S T$ aspartate aminotransferase, $E T$ endocrine therapy, ILD interstitial lung disease, $n$ number of patients, VTE venous thromboembolic event

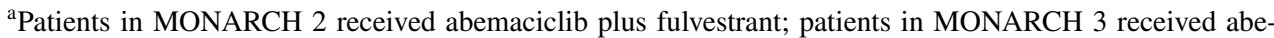
maciclib plus either letrozole or anastrozole

${ }^{\mathrm{b}}$ There were no Grade 4 diarrhea events

${ }^{\mathrm{c}}$ There were 3 fatal cases of VTEs in MONARCH 2 and 3, 2 in the $<65$ age group and 1 in the 65-74 age group

dAs used here, "ILD/Pneumonitis Events" is a consolidated term incorporating the MedDRA preferred terms "pneumonitis," "interstitial lung disease," "organising pneumonia," and "pulmonary fibrosis"

${ }^{\text {e}}$ There were 2 fatal cases in MONARCH 2, 1 in the $<65$ age group and 1 in the 65-74 age group. There was 1 fatal case in MONARCH 3, in the 65-74 age group dose adjustments and the use of antidiarrheals, antiemetics, and colony-stimulating factors (Table 5). The use of antidiarrheals and colony-stimulating factors was not different between age groups; however, there was an increase in the use of antiemetics in the 65-74 and $\geq 75$ groups compared to patients $<65$ years $(<65,11.6 \% ; 65-74,20.5 \% ; \geq 75,20.5 \%)$.

\section{Efficacy}

Consistent with the ITT population in MONARCH 2 and 3, PFS benefit with abemaciclib + ET was observed across age subgroups. (Fig. 2). There was no significant difference in abemaciclib treatment effect between the 3 age subgroups (MONARCH 2: interaction $p$-value, 0.695; MONARCH 3: interaction $p$-value, 0.634). In MONARCH 2 (Fig. 2a), estimated median PFS in the abemaciclib + ET arm compared to the placebo +ET arm was 17.4 months versus 10.8 
Table 5 AE management: pooled analysis of MONARCH 2 and MONARCH 3 dose adjustments, study treatment discontinuations, and concomitant medications

\begin{tabular}{|c|c|c|c|}
\hline & \multicolumn{3}{|c|}{ Abemaciclib +ET } \\
\hline & $<65(n=466)$ & $65-74(n=219)$ & $\geq 75(n=83)$ \\
\hline \multicolumn{4}{|l|}{ Dose adjustments, $n(\%)$} \\
\hline Pts with $\geq 1$ dose adjustment & $294(63.1)$ & $163(74.4)$ & $63(75.9)$ \\
\hline Pts with $\geq 1$ dose omission & $259(55.6)$ & $151(68.9)$ & $60(72.3)$ \\
\hline Dose omission due to AEs & $225(48.3)$ & $143(65.3)$ & $58(69.9)$ \\
\hline Diarrhea & $59(12.7)$ & 49 (22.4) & $25(30.1)$ \\
\hline Neutropenia & $75(16.1)$ & $46(21.0)$ & $8(9.6)$ \\
\hline Pts with $\geq 1$ dose reduction & $199(42.7)$ & $125(57.1)$ & $46(55.4)$ \\
\hline Dose reduction due to AEs & $175(37.6)$ & $120(54.8)$ & $46(55.4)$ \\
\hline Diarrhea & 68 (14.6) & $41(18.7)$ & $19(22.9)$ \\
\hline Neutropenia & $52(11.2)$ & $29(13.2)$ & $5(6.0)$ \\
\hline \multicolumn{4}{|l|}{ Study treatment discontinuations, $n(\%)$} \\
\hline Pts discontinued all study treatment due to $\mathrm{AE}$ & $41(8.8)$ & $31(14.2)$ & $20(24.1)$ \\
\hline Diarrhea & $2(0.43)$ & $4(1.83)$ & $4(4.82)$ \\
\hline Lung infection & $5(1.1)$ & $3(1.4)$ & $0(0.0)$ \\
\hline ALT increase & $4(0.9)$ & $3(1.4)$ & $0(0.0)$ \\
\hline \multicolumn{4}{|l|}{ Concomitant medications pts with $\geq 1, n(\%)$} \\
\hline Antidiarrheals & $342(73.4)$ & $157(71.7)$ & $60(72.3)$ \\
\hline Antiemetics & $54(11.6)$ & $45(20.5)$ & $17(20.5)$ \\
\hline G-CSF/GM-CSF & $30(6.4)$ & $14(6.4)$ & $2(2.4)$ \\
\hline
\end{tabular}

$A E$ adverse events, $A L T$ alanine amino transferase, ET endocrine therapy, $G$-CSF granulocyte colonystimulating factor, GM-CSF granulocyte/macrophage colony-stimulating factor, $n$ number of patients, Pts patients
(HR, $0.523 ; 95 \% \mathrm{CI}, 0.402-0.681)$ in the $<65$ age group, 14.4 months versus 8.1 (HR, 0.633; 95\% CI, 0.426-0.941) in 65-74 age group, and 13.9 months versus 5.8 (HR, 0.615; 95\% CI, 0.340-1.112) in the $\geq 75$ age group. In MONARCH 3 (Fig. 2b), median PFS in the abemaciclib + ET arm compared to the placebo +ET arm was 27.5 months versus 14.0 (HR, 0.480; 95\% CI, 0.346-0.666) in the $<65$ age group, 28.2 months versus 24.2 (HR, 0.635; 95\% CI, 0.395-1.020) in the 65-74 age group, and 31.1 months versus 9.1 (HR, 0.541 ; $95 \%$ CI, $0.258-1.134$ ) in the $\geq 75$ age group.

\section{Discussion}

This post hoc analysis of MONARCH 2 and 3 is the first to report age-specific safety, tolerability, and efficacy outcomes of abemaciclib + ET in women with $\mathrm{HR}+, \mathrm{HER} 2-\mathrm{ABC}$. By pooling the data across these two randomized, placebo-controlled, Phase 3 studies, we were able to increase the number of patients 75 years or older in our analysis, an age group that is more representative of a real-world geriatric population with respect to age. However, the proportion of patients in that demographic was still low, accounting for only $11.5 \%$ of the pooled safety population. Subjects enrolled in clinical trials may not be representative of the general population seen in clinical practice [5]. This is particularly true in the case of older patient populations, where narrow eligibility criteria often exclude all but the healthiest older patients [2]. MONARCH 2 and 3 studies were designed to explore safety in a broad age population; thus, the protocols did not include a comprehensive geriatric assessment for older patients. As a result, we are unable to fully characterize the fitness of the older patients included in this pooled analysis. More recently, the use of geriatric assessments in clinical trials enrolling older patients as standard practice has been suggested [21, 22]. The relevance of comprehensive geriatric assessments to guide CDK4 and 6 inhibitor treatment plans in older patients with breast cancer has also been noted [2].

There are also some limitations due to study differences: MONARCH 2 enrolled a more pretreated study population [16], and each study incorporated different ETs. Although outcome analysis and conclusions presented here apply to the abemaciclib dose approved in combination with ET (150 mg BID), the fact that MONARCH 2 initially enrolled patients on $200 \mathrm{mg}$ BID abemaciclib, which is the approved monotherapy dose [19], added some complexity to the safety analysis. Since the median number of days receiving $200 \mathrm{mg}$ of abemaciclib before dose reduction or discontinuation was 34 days, the impact was limited to toxicities that appear early in treatment, like diarrhea. Where possible, we have endeavored to point out instances where this higher dose may confound the interpretation of the pooled safety data. 


\section{(a) MONARCH 2}
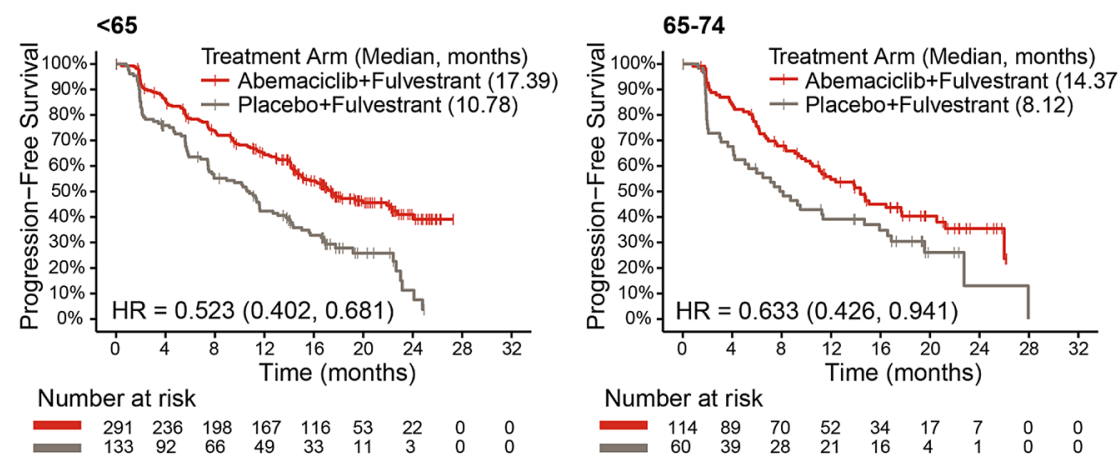

\section{(b) MONARCH 3}

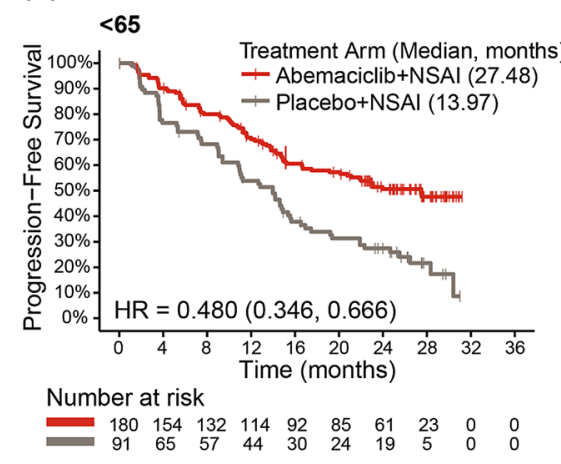

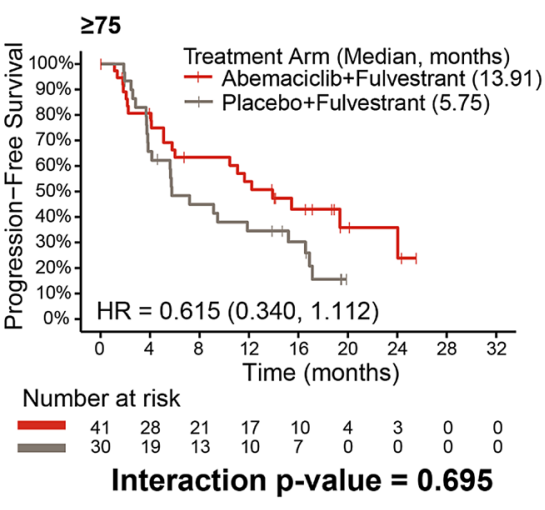

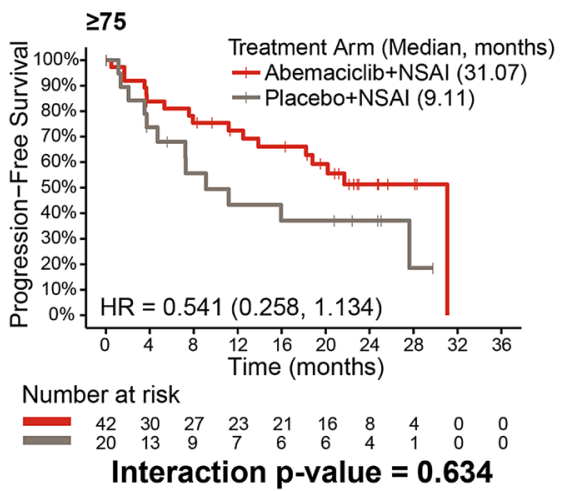

Fig. 2 PFS by age subgroup in a MONARCH 2 and b MONARCH 3. HR hazard ratio, NSAI nonsteroidal aromatase inhibitor

As expected with abemaciclib, the most frequent TEAEs were gastrointestinal toxicities. Nausea and decreased appetite were moderately increased in the 2 older subgroups, as was use of antiemetics. This is not unexpected considering patients in the 65-74 and $\geq 75$ age groups had a higher incidence of pre-existing gastrointestinal and metabolism and nutrition disorders that may have been aggravated by the exposure to abemaciclib. Although the incidence of any grade diarrhea was similar in patients treated with abemaciclib + ET across age groups, more patients age $\geq 75$ experienced clinically significant (Grade 2/3) diarrhea compared to the other age groups; the use of antidiarrheals was not different between age groups. Incidence of clinically significant diarrhea was highest in the $\geq 75$ age group regardless of treatment arm. Dose reductions due to diarrhea in the $\geq 75$ age group were influenced by the starting dose; $50 \%$ of patients starting at $200 \mathrm{mg}$ required dose reductions due to diarrhea compared to only $11 \%$ of patients starting at $150 \mathrm{mg}$. Therefore, while the overall safety findings were consistent across the pre-amendment and post-amendment populations, there were some differences in toxicities expected to occur early in the course of treatment such as diarrhea and other gastrointestinal toxicities.
Neutropenia is also a frequent TEAE associated with abemaciclib and other CDK4 and 6 inhibitors [23]. In our pooled analysis, neutropenia was the most common Grade $\geq 3 \mathrm{AE}$; interestingly, incidence of neutropenia was not increased in patients $>65$ years, consistent with data reported from an FDA pooled analysis of CDK4 and 6 inhibitors in older women [5]. In fact, in our analysis, the $\geq 75$ age group had the lowest incidence of neutropenia; this was also reflected by the lower rate of G-CSF/GM-CSF use in this age group.

VTEs are a known AE of special interest for abemaciclib. The incidence of VTE was higher in all age groups in the abemaciclib arm compared to the placebo arm in MONARCH 2 and 3. Of note, VTEs are not specific to abemaciclib but have been reported for CDK inhibitors as a class effect [24]. The higher incidence of VTE in the subgroup of patients $\geq 75$, together with age being a risk factor, suggests that these patients should be more carefully monitored for early symptoms. A recent review by the FDA suggests that the occurrence of ILD/pneumonitis may also be a class effect [25]; rates of ILD/pneumonitis reported in MONARCH 2 and 3 are similar to those observed in studies of other CDK4 and 6 inhibitors. In our pooled analysis, ILD/pneumonitis 
was reported at similar frequency $(\sim 3.5 \%)$ across age groups in the abemaciclib + ET arm.

While abemaciclib (150 mg BID) + ET demonstrated a generally tolerable safety profile in older patients, with no new safety concerns compared to the overall MONARCH 2 and 3 populations $[15,16]$, higher rates of some AEs occurred in older patients. Patients in the 65-74 and $\geq 75$ age groups were more likely to have had potentially confounding comorbidities including hypertension, gastrointestinal disorders, and metabolism/nutritional disorders. In addition, there were more patients with ECOG PS 1 in the two older subgroups; however, no other assessments were done to further characterize the fitness/frailty in the older patients, and we acknowledge that ECOG PS is not the best indicator of functional impairment in this population [26, 27]. Patients in the 2 older subgroups required more dose adjustments to manage AEs, which is in line with a previously published analysis of CDK4 and 6 inhibitors [5]. Of note, dose adjustment data from the pooled analysis were impacted by starting dose in MONARCH 2, especially the $>75$ age group that had the highest proportion of patients starting on the $200 \mathrm{mg}$ dose (34.1\%). The most frequent $\mathrm{AE}$ causing dose reductions was diarrhea, and the $\geq 75$ subgroup had a modest increase (8\%) over the $<65$ group. This could possibly be due to a more conservative management of the toxicities in older patients because of their additional burden of comorbidities and the clinical impact that even low-grade toxicities may have in these populations [28].

Abemaciclib + ET provided a consistent PFS benefit across all age groups with a clinically relevant magnitude of effect observed in all age groups as indicated by the lack of significance in the interaction $p$ values. Overall, median PFS in patients receiving ET-only was shorter in older patients. For instance, in MONARCH 2, median PFS in the placebo arm was much lower in the older age group relative to the younger age group: 10.78 months in patients $<65$ versus 5.75 months in patients $\geq 75$ years. In MONARCH 3 , PFS in the placebo arm was also lower in older versus younger age groups but to a lesser extent: 13.97 months in patients $<65$ versus 9.11 months in patients $\geq 75$ years. However, in patients 65-74 years old, median PFS in the placebo arm was much longer (24.20 months). Our analysis shows that the addition of abemaciclib to the ET backbone provides a PFS benefit across all age groups, including in the two older age groups. More research is warranted to better characterize the impact of age on safety and efficacy outcomes, using end points more relevant for older patients such as quality of life and maintenance of functional status [21]. Notably, a prospective phase II study (NCT04305834) is ongoing and designed to specifically estimate the incidence of grade 3 or higher toxicities attributed to abemaciclib monotherapy in adults aged 70 or older with HR+ metastatic breast cancer [29].
Taken together, our data suggest that while abemaciclib was generally well tolerated overall, clinicians should be aware of potentially higher incidence of gastrointestinal toxicities in older patients, including Grade $2 / 3$ diarrhea. A proactive approach to treating older patients should include more careful monitoring of toxicities, including low grade adverse events, dose adjustments and use of supportive medication for gastrointestinal toxicities with the goal of maximizing abemaciclib tolerability. While age appears to be an important factor associated with higher rates of toxicity, age alone should not be considered in isolation when making treatment decisions. Future trials are warranted to further characterize the safety and efficacy of abemaciclib and other CDK4 and 6 inhibitors in broader older patient populations that are more representative of real-world settings.

Acknowledgements We thank the patients, caregivers, investigators, and support staff who were involved in the MONARCH clinical trials. Sarah Cassidy Nabinger of Eli Lilly and Company provided project management support. Medical writing and editorial support were provided by Erika Wittchen and Dana Schamberger of Syneos Health, and funded by Eli Lilly and Company. Fulvestrant (Faslodex ${ }^{\circledR}$ ) was provided by AstraZeneca for the MONARCH 2 trial.

Authors contribution All authors contributed to at least one of the following: study conception, design, data acquisition, analysis, and/ or interpretation. All authors contributed to drafting of the manuscript and/or critical revision of the work for important intellectual content. All authors read and approved the final manuscript.

Funding This study was funded by Eli Lilly and Company.

\section{Compliance with ethical standards}

Conflict of interest Funding M.P. Goetz reports research funding from the National Cancer Institute, National Institutes of Health, Department of Defense, Mayo Development, Eli Lilly, Pfizer, Alliance Foundation Trials, Sermonix Pharmaceutical, Translational Breast Cancer Research Consortium, and Minnesota Partnership for Biotechnology and Medical Genomics. H. Wildiers reports consulting fees and honoraria from AstraZeneca, Biocartis, Lilly, Novartis, Pfizer, PUMA Biotechnology, Roche, Sirtex, and Daiiji (all to institution); research grants from Roche and Novartis (all to institution); and travel support from Roche and Pfizer (to H.W). M. Campone received travel support from Pfizer, Novartis, Roche, and AstraZeneca. L. Manso reports consulting fees from Roche, Astra, Novartis, Tesaro, and Pfizer. M. Toi reports research funding from Takeda, Chugai, Pfizer, Taiho, Kyowa-Kirin, Eisai, JBCRG association, Daiichi-Sankyo, AstraZeneca, Astellas, Shimadzu, Nippon Kayaku, and AFI technology. G. Sledge Jr. reports research support from Eli Lilly and Company; research support from Pfizer. Financial interests M.P. Goetz reports consulting fees (all to institution) from Eagle Pharmaceuticals, Eli Lilly, Biovica, Novartis, Sermonix, Context Pharm, Pfizer, and Biotheranostics. M. Toi reports consultation fees from Kyowa-Kirin, Daiichi-Sankyo, Konica-Minolta, BMS, Athenex Oncology, and Bertis; and lecture fees from Takeda, Chugai, Eli Lilly Pfizer, Taiho, Kyowa-Kirin, Eisai, Daiichi-Sankyo, MSD, AstraZeneca, Astellas, Shimadzu, Genomic Health, Novartis, Yakult, and Nippon Kayaku. V.A-M. André, N. Chouaki, and B. San Antonio report stocks/shares in Eli Lilly and Company. Non-financial interests M.P. Goetz has consulted for or is/was a board member of NCI Breast Cancer Steering Committee, Translational Breast Cancer 
Research Consortium, NCCN Breast Cancer Panel, Clinical Pharmacogenetics Implementation Consortium Guide for CYP2D6 and Tamoxifen, NCI SPORE Special Emphasis Panel, and Academic and Community Cancer Research United. M. Campone has consulted for Pierre Fabre Oncology, Sanofi, Novartis, and Servier; speakers' bureau, Novartis. M. Toi has served on Editorial Boards for Breast Cancer Research and Treatment, Scientific Reports, Frontier Oncology, Cancer Science, International Journal of Clinical Oncology, Asian Journal of Surgery, and International Journal of Biological Markers; and is/ was a member of the Board of Directors for Japanese Breast Cancer Research group association, Kyoto Breast Cancer Research network, and Organisation for Oncology and Translational Research. G. Sledge Jr. reports consulting for Syndax, Symphogen, Verseau Therapeutics, and Eli Lilly and Company; Board of Directors for Tessa Therapeutics. Employment V.A-M. André, N. Chouaki, and B. San Antonio are employees of Eli Lilly and Company. M. Okera declares no competing interests. E-M. Grischke declares no competing interests.

Research involving human participants and/or animals Both studies were reviewed and approved by ethical and institutional review boards. The trials were conducted in accordance with the 1964 Declaration of Helsinki and its later amendments or comparable ethical standards. This article does not contain any studies with animals performed by any of the authors.

Informed consent Informed consent was obtained from all individual participants included in the study.

Data availability All data generated or analyzed during this study are included in this published article.

Open Access This article is licensed under a Creative Commons Attribution 4.0 International License, which permits use, sharing, adaptation, distribution and reproduction in any medium or format, as long as you give appropriate credit to the original author(s) and the source, provide a link to the Creative Commons licence, and indicate if changes were made. The images or other third party material in this article are included in the article's Creative Commons licence, unless indicated otherwise in a credit line to the material. If material is not included in the article's Creative Commons licence and your intended use is not permitted by statutory regulation or exceeds the permitted use, you will need to obtain permission directly from the copyright holder. To view a copy of this licence, visit http://creativecommons.org/licenses/by/4.0/.

\section{References}

1. SEER cancer statistics: common cancer sites (2019) National Cancer Institute. https://seer.cancer.gov/statfacts/html/common.html

2. Battisti NML, De Glas N, Sedrak MS, Loh KP, Liposits G, SotoPerez-de-Celis E, Krok-Schoen JL, Menjak IB, Ring A (2018) Use of cyclin-dependent kinase 4/6 (CDK4/6) inhibitors in older patients with ER-positive HER2-negative breast cancer: Young International Society of Geriatric Oncology review paper. Ther Adv Med Oncol 10:1-26. https://doi.org/10.1177/1758835918 809610

3. Cancer stat facts: female breast cancer (2019) National Cancer Institute, SEER Program. https://seer.cancer.gov/statfacts/html/ breast.html

4. Howlader N, Altekruse SF, Li CI, Chen VW, Clarke CA, Ries LA, Cronin KA (2014) US incidence of breast cancer subtypes defined by joint hormone receptor and HER2 status. J Nat Cancer Inst. https://doi.org/10.1093/jnci/dju055
5. Howie LJ, Singh H, Bloomquist E, Wedam S, Amiri-Kordestani L, Tang S, Sridhara R, Sanchez J, Prowell TM, Kluetz PG, KingKallimanis BL, Gao JJ, Ibrahim A, Goldberg KB, Theoret M, Pazdur R, Beaver JA (2019) Outcomes of older women with hormone receptor-positive, human epidermal growth factor receptornegative metastatic breast cancer treated with a CDK4/6 inhibitor and an aromatase inhibitor: an FDA pooled analysis. J Clin Oncol 37(36):3475-3483. https://doi.org/10.1200/JCO.18.02217

6. Scotté F, Bossi P, Carola E, Cudennec T, Dielenseger P, Gomes F, Knox S, Strasser F (2018) Addressing the quality of life needs of older patients with cancer: a SIOG consensus paper and practical guide. Ann Oncol 29(8):1718-1726. https://doi.org/10.1093/ annonc/mdy 228

7. Singh H, Hurria A, Klepin HD (2018) Progress through collaboration: an ASCO and U.S. Food and Drug Administration workshop to improve the evidence base for treating older adults with cancer. 2018 ASCO educational book

8. Riseberg D (2015) Treating elderly patients with hormone receptor-positive advanced breast cancer. Clin Med Insights Oncol 9:65-73. https://doi.org/10.4137/cmo.s26067

9. Savard M-F, Khan O, Hunt KK, Verma S (2019) Redrawing the lines: the next generation of treatment in metastatic breast cancer. Am Soc Clin Oncol Educ Book 39:e8-e21. https://doi. org/10.1200/edbk_237419

10. NCCN Clinical Practice Guidelines in Oncology: breast cancer (Version 2.2020) (2020). National Comprehensive Cancer Network (NCCN). https://www.nccn.org/professionals/physician_gls/ pdf/breast_blocks.pdf

11. Cardoso F, Senkus E, Costa A, Papadopoulos E, Aapro M, André F, Harbeck N, Aguilar Lopez B, Barrios CH, Bergh J, Biganzoli L, Boers-Doets CB, Cardoso MJ, Carey LA, Cortés J, Curigliano G, Diéras V, El Saghir NS, Eniu A, Fallowfield L, Francis PA, Gelmon K, Johnston SRD, Kaufman B, Koppikar S, Krop IE, Mayer M, Nakigudde G, Offersen BV, Ohno S, Pagani O, Paluch-Shimon S, Penault-Llorca F, Prat A, Rugo HS, Sledge GW, Spence D, Thomssen C, Vorobiof DA, Xu B, Norton L, Winer EP (2018) 4th ESO-ESMO international consensus guidelines for advanced breast Cancer (ABC 4). Ann Oncol 29(8):1634-1657. https://doi.org/10.1093/annonc/mdy192

12. Giuliano M, Schettini F, Rognoni C, Milani M, Jerusalem G, Bachelot T, De Laurentiis M, Thomas G, De Placido P, Arpino G, De Placido S, Cristofanilli M, Giordano A, Puglisi F, Pistilli B, Prat A, Del Mastro L, Venturini S, Generali D (2019) Endocrine treatment versus chemotherapy in postmenopausal women with hormone receptor-positive, HER2-negative, metastatic breast cancer: a systematic review and network meta-analysis. Lancet Oncol 20(10):1360-1369. https://doi.org/10.1016/s1470-2045(19)30420 $-6$

13. Eggersmann TK, Degenhardt T, Gluz O, Wuerstlein R, Harbeck $\mathrm{N}$ (2019) CDK4/6 inhibitors expand the therapeutic options in breast cancer: palbociclib, ribociclib and abemaciclib. Biodrugs 33(2):125-135. https://doi.org/10.1007/s40259-019-00337-6

14. Marra A, Curigliano G (2019) Are all cyclin-dependent kinases 4/6 inhibitors created equal? NPJ Breast Cancer 5(1):27. https:// doi.org/10.1038/s41523-019-0121-y

15. Goetz MP, Toi M, Campone M, Sohn J, Paluch-Shimon S, Huober J, Park IH, Tredan O, Chen SC, Manso L, Freedman OC, Garnica Jaliffe G, Forrester T, Frenzel M, Barriga S, Smith IC, Bourayou N, Di Leo A (2017) MONARCH 3: abemaciclib as initial therapy for advanced breast cancer. J Clin Oncol 35(32):3638-3646. https ://doi.org/10.1200/jco.2017.75.6155

16. Sledge GWJ, Toi M, Neven P, Sohn J, Inoue K, Pivot X, Burdaeva O, Okera M, Masuda N, Kaufman PA, Koh H, Grischke E-M, Frenzel M, Lin Y, Barriga S, Smith IC, Bourayou N, LlombartCussac A (2017) MONARCH 2: abemaciclib in combination with fulvestrant in women with HR+/HER2- advanced breast 
cancer who had progressed while receiving endocrine therapy. J Clin Oncol 35(25):2875-2884. https://doi.org/10.1200/ jco.2017.73.7585

17. Johnston S, Martin M, Di Leo A, Im S-A, Awada A, Forrester T, Frenzel M, Hardebeck MC, Cox J, Barriga S, Toi M, Iwata H, Goetz MP (2019) MONARCH 3 final PFS: a randomized study of abemaciclib as initial therapy for advanced breast cancer. NPJ Breast Cancer 5(1):5. https://doi.org/10.1038/s41523-018-0097-z

18. Sledge GW Jr, Toi M, Neven P, Sohn J, Inoue K, Pivot X, Burdaeva O, Okera M, Masuda N, Kaufman PA, Koh H, Grischke EM, Conte P, Lu Y, Barriga S, Hurt K, Frenzel M, Johnston S, Llombart-Cussac A (2019) The effect of abemaciclib plus fulvestrant on overall survival in hormone receptor-positive, ERBB2-negative breast cancer that progressed on endocrine therapy-MONARCH 2: a randomized clinical trial. JAMA Oncol 6(1):116-124. https://doi.org/10.1001/jamaoncol.2019.4782

19. Dickler MN, Tolaney SM, Rugo HS, Cortes J, Dieras V, Patt D, Wildiers H, Hudis CA, O'Shaughnessy J, Zamora E, Yardley DA, Frenzel M, Koustenis A, Baselga J (2017) MONARCH 1, a phase II study of abemaciclib, a CDK4 and CDK6 inhibitor, as a single agent, in patients with refractory HR(+)/HER2(-) metastatic breast cancer. Clin Cancer Res 23(17):5218-5224. https://doi. org/10.1158/1078-0432.ccr-17-0754

20. Rugo HS, Huober J, Garcia-Saenz JA, Masuda N, Sohn JH, Andre VAM, Barriga S, Cox J, Goetz M (2020) Management of abemaciclib-associated adverse events in patients with hormone receptorpositive, human epidermal growth receptor 2-negative advanced breast cancer: safety analysis of MONARCH 2 and MONARCH 3. Oncologist Epub ahead of print. doi:https://doi.org/10.1002/ onco. 13531

21. Soto-Perez-De-Celis E, Lichtman SM (2017) Considerations for clinical trial design in older adults with cancer. Expert Opin Investig Drugs 26(10):1099-1102. https://doi.org/10.1080/13543 784.2017.1369043

22. Wildiers H, de Glas NA (2020) Anticancer drugs are not well tolerated in all older patients with cancer. Lancet Healthy Longev 1:e43-47. https://doi.org/10.1016/S2666-7568(20)30001-5

23. Spring LM, Wander SA, Zangardi M, Bardia A (2019) CDK $4 / 6$ inhibitors in breast cancer: current controversies and future directions. Curr Oncol Rep 21(3):25. https://doi.org/10.1007/ s11912-019-0769-3

24. Thein K, Zaw M, Tun A, Jones C, Radhi S, Hardwicke F, Oo T (2018) Abstract P3-14-02: incidence of venous thromboembolism in patients with hormone receptor-positive HER2-negative metastatic breast cancer treated with CDK 4/6 inhibitors: a systematic review and meta- analysis of randomized controlled trials. Cancer Res 78(4 Supplement):P3-14-02-P13-14-02. doi:https://doi. org/10.1158/1538-7445.sabcs17-p3-14-02

25. FDA warns about rare but severe lung inflammation with Ibrance, Kisqali, and Verzenio for breast cancer (2019) U.S. food and drug administration. https://www.fda.gov/drugs/drug-safety-and-avail ability/fda-warns-about-rare-severe-lung-inflammation-ibrancekisqali-and-verzenio-breast-cancer

26. Wildiers H, Heeren P, Puts M, Topinkova E, Janssen-Heijnen ML, Extermann M, Falandry C, Artz A, Brain E, Colloca G, Flamaing J, Karnakis T, Kenis C, Audisio RA, Mohile S, Repetto L, Van Leeuwen B, Milisen K, Hurria A (2014) International Society of Geriatric Oncology consensus on geriatric assessment in older patients with cancer. J Clin Oncol 32(24):2595-2603. https://doi. org/10.1200/jco.2013.54.8347

27. Simcock R, Wright J (2020) Beyond performance status. Clin Oncol (Royal College of Radiologists) 32(9):553-561. https://doi. org/10.1016/j.clon.2020.06.016

28. Kalsi T, Babic-Illman G, Fields P, Hughes S, Maisey N, Ross P, Wang Y, Harari D (2014) The impact of low-grade toxicity in older people with cancer undergoing chemotherapy. Br J Cancer 111(12):2224-2228. https://doi.org/10.1038/bjc.2014.496

29. Clinicaltrials.gov. Abemaciclib monotherapy in treating older patients with hormone receptor positive metastatic breast cancer (NCT04305834). Available at https://clinicaltrials.gov/ct2/show/ NCT04305834. Accessed October 302020

Publisher's Note Springer Nature remains neutral with regard to jurisdictional claims in published maps and institutional affiliations. 